\title{
LEIOMIOMA BENIGNO METASTATIZANTE DE VEIA CAVA INFERIOR: RARA COMPLICAÇÃO TARDIA DE HISTERECTOMIA
}

\author{
BENIGN METASTASIZING LEIOMYOMA OF INFERIOR VENA CAVA: A RARE \\ CONDITION FOLLOWING HYSTERECTOMY
}

\author{
Marcos Filgueiras, TCBC - RJ'1; Antônio Carlos Barros Lima Jr. ${ }^{2}$; Walter Giulliano Baldoni³; \\ Washington Sérgio Gonçalves Milezi, TCBC-RJ ${ }^{4}$; Ricardo Zorron, TCBC - RJ ${ }^{5}$
}

\section{INTRODUÇÃO}

Tumores de grandes vasos são classicamente difíceis de diagnosticar, pela dificuldade de interpretar exames de imagens que consigam delimitar corretamente os tumores em seu sítio e também devido à pouca sintomatologia apresentada quando estes ainda não obstruíram o vaso por completo.

O leiomioma benigno metastatizante (LBM) é uma afecção rara com aproximadamente 75 casos relatados na literatura científica mundial, sendo seu sítio mais comum o pulmão ${ }^{1}$. Este termo foi cunhado por Steiner em 1939, em relato de caso de paciente de 36 anos que morreu após um elevado número de metástases pulmonares e linfáticas hilares oriundas de um leiomioma uterino² ${ }^{2}$ Há na literatura, relatos esporádicos sobre leiomioma metastatizante com implantação na veia cava inferior.

O LBM de veia cava pode ocorrer como conseqüência de um leiomioma uterino em pacientes com a doença ativa ou, mais freqüentemente, naquela com história pregressa de histerectomia. Não há associação com fertilidade ou paridade da paciente. Seu diagnóstico pré-operatório é muito difícil, somente podendo ser feito com os achados intra-operatórios e o posterior exame anatomopatológico da peça. Devido à dificuldade de identificação, deve-se ter em mente que os LBM são subdiagnosticados ${ }^{1,3}$.

\section{RELATO DO CASO}

Paciente do sexo feminino, 54 anos, portadora de hipertensão arterial sistêmica em tratamento regular com história familiar para doenças cardiovasculares. Possuía história prévia de colecistectomia videolaparoscópica há três anos e histerectomia parcial há 16 meses devido à miomatose, com útero que se mostrava de textura heterogênea, com nódulos pequenos hipoecóicos intramurais fúndicos, um deles medin- do $1,5 \mathrm{~cm} \times 2 \mathrm{~cm}$ e o outro, calcificado, medindo $2,5 \mathrm{~cm} \times 2,4 \mathrm{~cm}$. Há um ano procurou atendimento médico para avaliação de esteatose hepática, descoberta através de ultrassonografia (USG) pré-operatória para colecistectomia, realizada três anos antes, que não demonstrava lesão nos grandes vasos. Relatava desconforto abdominal leve como única sintomatologia. Seu exame físico não apresentava alterações. Na USG abdominal de admissão evidenciou-se nodulação hipoecóica no hipocôndrio direito, de contornos regulares e textura homogênea entre o rim direito e a veia cava, acima do músculo psoas, medindo $7,5 \mathrm{~cm} \times 3,5 \mathrm{~cm} \times 4 \mathrm{~cm}$. Foi realizada tomografia computadorizada (TC) abdominal que evidenciava massa retroperitoneal à direita, sem plano de clivagem com a veia cava e com o músculo psoas direito. A ressonância magnética nuclear (RMN) apresentava formação expansiva infiltrante, isointensa em T1 e T2 de limites pouco definidos, captando levemente o meio de contraste, aparentemente com limites com a segunda e terceira porções duodenais, promovendo espessamento parietal destes segmentos. Foi indicada laparotomia exploradora, sendo realizada ressecção da massa com parte da parede lateral da veia cava inferior por clampeamento lateral e sutura contínua com Prolene ${ }^{\circledR} 4.0$ (Figuras. 1 e 2).

O exames histopatológicos macroscópico e microscópico evidenciaram leiomioma benigno metastatizante vascular com células tumorais isoladas e dispersas, ausência de necrose, hemorragia e atividade mitótica nas peças estudadas. Além disso, o estudo imunohistoquimico demonstrou alta positividade para desmina, alfa-actina de músculo liso e receptor de progesterona, sendo negativo para proteína S100, CD34 e receptor de estrogênio. JAUTZKE et al relataram cinco casos de LBM onde quatro eram positivos para receptores de estrogênio e todos os cinco eram positivos para receptores de progesterona ${ }^{3}$. A paciente teve boa evolução pós-operatória e encontra-se assintomática após 24 meses.

1. Chefe do Serviço de Cirurgia- HCTCO - Faculdade de Medicina de Teresópolis- FESO; Professor Adjunto da Cadeira de Cirurgia - Faculdade de Medicina de Teresópolis - FESO.

2. Cirurgião do Serviço de Cirurgia - HCTCO - Faculdade de Medicina de Teresópolis - FESO.

3. Cirurgião Vascular- HCTCO - Faculdade de Medicina de Teresópolis - FESO. Cirurgião Vascular- Hospital Geral de Bonsucesso.

4. Cirurgião do Serviço de Cirurgia- HCTCO - Faculdade de Medicina de Teresópolis -FESO; Professor Assistente da Cadeira de Cirurgia - Faculdade de Medicina de Teresópolis - FESO.

5. Professor Titular e Regente da Cadeira de Cirurgia - Faculdade de Medicina de Teresópolis - FESO; Cirurgião do Hospital Municipal Lourenço Jorge - Rio de Janeiro - RJ.

Trabalho realizado no Serviço de Cirurgia - Hospital das Clínicas Constantino Otaviano- HCTCO e Disciplina de Clínica Cirúrgica da Faculdade de Medicina de Teresópolis, FESO, Teresópolis, Rio de Janeiro. 


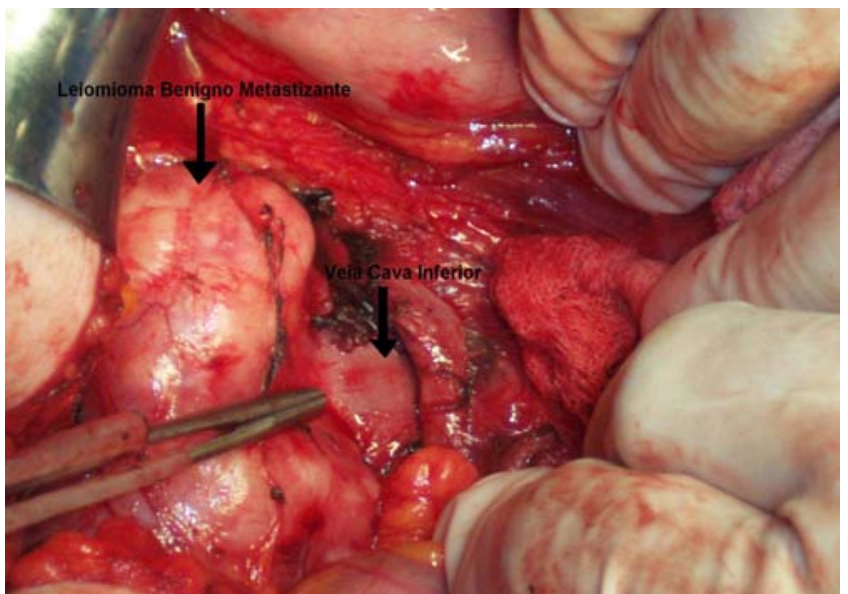

Figura 1 - Aspecto intra-operatório do LBM intravascular de veia cava inferior.

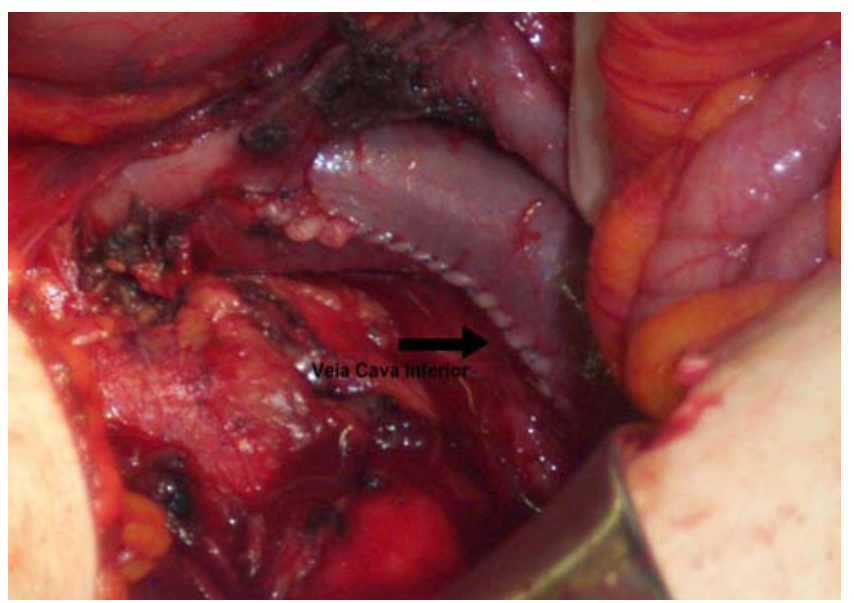

Figura 2 - Sutura lateral após ressecção de fragmento de veia cava inferior.

\section{DISCUSSÃO}

Os LBM são tumores raros encontrados em mulheres com historia pregressa de histerectomia por leiomioma uterino, podendo ser achados desde três meses até 20 anos após a cirurgia ${ }^{1,3,4,5}$. Kremer listou entre 1868 e 1975 apenas 11 casos de leiomioma benigno metastatizante vascular, sendo sua localização mais comum na veia safena ${ }^{5}$. Jautzke et al em 1996 revisaram 74 casos de LBM e reportaram o sítio pulmonar como a localização mais comum destas lesões. Os LBM são mais comuns no pulmão, seguido de coração, ossos, cérebro e vasos sanguíneos ${ }^{3,5}$. A aparência radiológica pulmonar é indistinguível de uma metástase maligna pulmonar. Assim, a confirmação deve ser feita com "core biopsy " ou punção percutânea com agulha fina ${ }^{1}$.

A patogênese também é objeto de controvérsia e, ainda que várias teorias tenham sido propostas, a disseminação vascular é a mais largamente aceita ${ }^{1,2,5}$. A condição necessária ao diagnóstico histológico do leiomioma metastatizante com invasão vascular é a proliferação de células musculares lisas neste sítio, em pacientes com história pregressa ou atual de leiomioma uterino ${ }^{1,5}$. São tumores assintomáticos, porém em períodos avançados podem apresentar dispnéia e tosse, nas metástases pulmonares, edema em membros inferiores, oclusão completa da veia cava inferior e sintomas de insuficiência cardíaca quando há extensão para o coração ${ }^{4}$. Pacientes com LBM possuem história pregressa de leiomioma uterino, tratado com histerectomia, curetagem ou miomectomia. As pacientes podem possuir sintomas compatíveis com leiomioma uterino no momento da investigação, devido a não prévia identificação deste, dentre esses, os mais comuns são distúrbios menstruais (80\%), dismenorréia e sangramentos vultuosos. Há ainda casos em que a descoberta do LBM foi prévia a da afecção uterina.

O diagnóstico diferencial deve feito com leiomiosarcoma de veia cava, estimado entre 0,13 a $6 \%$, usualmente associado com células gigantes tipo osteoclastos, trombose parcial de veia cava inferior, com leiomiomatose intravascular de veia cava ${ }^{5}$. Pode ser observada a Síndrome de Budd-Chiari quando ocorrer um envolvimento superior da veia cava. A maioria dos LBM é positiva para receptores de estrogênio e receptores de progesteronas, associado à alta reatividade da desmina e alfa-actina do músculo liso, sendo o prognóstico da metástase benigna do leiomioma dependente do status do seu receptor de estrógeno ${ }^{1,3,4,5}$.

O tratamento básico para o LBM é a sua extirpação cirúrgica, completa, havendo consenso em realizar histerectomia simultânea à ressecção das massas extra-uterinas. Quando o LBM é irressecável opta-se pela inibição medicamentosa por uso de tamoxifeno, danazol com gestrina e agonista $\mathrm{GnRh}$ com progesterona, pela ooforectomia, ou com o uso de radioterapia, sendo a primeira delas a mais aceita pela sua reversibilidade e a mais eficaz contra as metástases benignas do pulmão. $\mathrm{O}$ acompanhamento da evolução pode ser feito através da ultrasonografia ou RMN a cada seis meses ${ }^{1,4}$.

Em resumo, o leiomioma benigno metastatizante vascular é uma afecção rara e de difícil diagnóstico diferencial, e deve-se cogitar o diagnóstico em pacientes com massa vascular e história prévia de cirurgia para miomatose uterina.

\footnotetext{
ABSTRACT

Large vessel tumors diagnosis is usually difficult. Benign metastasizing leiomyoma is a rare condition occurring inside peripheral vessels. There are few publications about this tumor inside inferior vena cava. A 54 years old female patient is presented with a previous hysterectomy for myomas. She complained of no specific symptoms 18 months after surgery. Computer tomography revealed a $7.5 \times 3.5 \times 4.0 \mathrm{~cm}$ mass at inferior vena cava and right psoas muscle. After surgical management and immunohistochemical screening biopsy, the diagnosis was benign metastasizing leiomyoma of inferior vena cava. The patient recovery was uneventful. Benign metastasizing leiomyoma of inferior vena cava is a very rare condition, and must be suspected in patients with primary leiomyosarcoma, especially in women previously submitted to hysterectomy for leiomyomatosis (Rev. Col. Bras. Cir. 2008; 35(4): 274-276).
}

Key words: Leiomyoma/complications; Vena cava, inferior; Hysterectomy. 


\section{REFERÊNCIAS}

1. Abramson S, Gilkeson RC, Goldstein JD, Woodard PK, Eisenberg $\mathrm{R}$, Abramson N. Benign metastasizing leiomyoma: clinical, imaging, and pathologic correlation. AJR Am J Roentgenol. 2001;176(6):1409-13.

2. Steiner PE. Metastasizing fibroleiomyoma of the uterus. Am J Pathol. 1939;15(1):89-109.

3. Jautzke G, Müller-Ruchholtz E, Thalmann U. Immunohistological detection of estrogen and progesterone receptors in multiple and well differentiated leiomyomatous lung tumors in women with uterine leiomyomas. Pathol Res Pract. 1996;192(3):215-23.

4. Koh DM, Burn PR, King DM. Benign metastasizing leiomyoma with intracaval leiomyomatosis. Br J Radiol. 2000;73(868):435-7.

5. Nam MS, Jeon MJ, Kim YT, Kim JW, Park KH, Hong YS. Pelvic leiomyomatosis with intracaval and intracardiac extension: a case report and review of the literature. Gynecol Oncol. 2003;89(1):175-80.
Como citar este artigo:

Filgueiras M, Lima Jr. ACB, Baldoni WG, Milezi WSG, Zorrón R. Leiomioma benigno metastatizante de veia cava inferior: rara complicação tardia de histerectomia. Rev Col Bras Cir. [periódico na Internet] 2008; 35(4). Disponível em URL: http://www.scielo.br/rcbc

Endereço para Correspondência:

Dr. Marcos Filgueiras

Rua Prefeito SebastiãoTeixeira, 20 - sala 505

25950 -Teresópolis - RJ.

E-mail: filgueirasmarcos@terra.com.br 